Pacific Journal of Mathematic 


\title{
THE BOREL PROPERTY OF SUMMABILITY METHODS
}

\author{
J. D. HIL L
}

1. Introduction. Let $T$ denote the method of summability corresponding to the real matrix $\left(a_{n, k}\right)$, for the moment arbitrary, by means of which a sequence $\left\{s_{k}\right\}$ is said to be summable- $T$ to $s$ if each of the series in

$$
t_{n}=\sum_{k=1}^{\infty} a_{n, k} s_{k} \quad(n=1,2,3, \cdots),
$$

is convergent and if $t_{n} \longrightarrow s$.

We shall be concerned here exclusively with the class $\mathscr{X}$ of all sequences $x=$ $\left\{\alpha_{k}\right\}$ where the $\alpha_{k}$ are 0 or 1 with infinitely many l's. A biunique mapping of the class $X$ into the real interval $\mathbb{N} \equiv(0<y \leq 1)$ is obtained by defining $y$ as the dyadic fraction $0 . \alpha_{1} \alpha_{2} \alpha_{3} \cdots$ corresponding to $x=\left(\alpha_{1}, \alpha_{2}, \alpha_{3}, \cdots\right)$, and conversely. This enables us to employ the phrase, "almost all sequences of 0 's and 1 's," by which is meant a subset of $\mathfrak{X}$ for which the corresponding subset of $\mathfrak{Y}$ has Lebesgue measure one.

A classical result of Borel [2] may be interpreted as asserting that almost all sequences of 0 's and 1 's are summable- $(C, 1)$, Cesàro of order one, to the value $1 / 2$. If the corresnonding statement is true for the method $T$ defined by (1.1) we shall say that $T$ has the Borel property, or more briefly, that $T \in(B P)$.

A study of the Borel property for regular methods $T$ was undertaken recently by the author $[5]$. In the present paper we dispense with the assumption of regularity, and in $\$ 2$ we investigate the consequences of assuming merely that $T \in$ $(B P)$. Two independent necessury conditions, (2.2) and (2.5), are obtained.

In $\$ 3$ it is shown by means of an cxample due essentially to Erdös that these conditions are not sufficient in order that $T \in(B P)$ even if condition (2.10) is added. By virtue of a lemma of Khintchine we are able to state in Theorem (3.5) a new sufficient condition considerably weaker than that given in Theorem (2.14) of [5]. For comparison the latter result is repeated here in Theorem (3.3). In Theorem

Received February 17, 1951.

Pacific J. Math. 1 (1951), 399-409. 
(3.11) we deal with a conjecture of Erdös and prove incidentally that in general the Borel property does not depend on the rate at which $\sum_{k=1}^{\infty} a_{n, k}^{2}$ approaches zero.

At the present time it appears unlikely that the Borel property can be characterized in any reasonably simple manner, at least if no restrictions are imposed on the matrix $\left(a_{n, k}\right)$ at the outset. This aspect of the problem remains to be considered.

2. Necessary conditions. We shall establish the following result.

(2.1) THEOREM. In order that $T \in(B P)$ the following conditions are necessary:

$$
\sum_{k=1}^{\infty} a_{n, k} \text { converges for each } n \text { and tends to } 1 \text { as } n \longrightarrow \infty \text {; }
$$

$$
A_{n} \equiv \sum_{k=1}^{\infty} a_{n, k}^{2}<\infty \text { for each } n
$$

$$
\lim _{n \rightarrow \infty} a_{n, k}=0 \text { for each } k
$$

$$
\lim _{n \rightarrow \infty} A_{n}=0
$$

Proof. If $T \in(B P)$, there exists a subset $\mathfrak{Y}^{*}$ of $\mathfrak{Y} \equiv(0<y \leq 1)$ of measure one such that

$$
t_{n}(y) \equiv \sum_{k=1}^{\infty} a_{n, k} a_{k}(y)
$$

is defined for each $n$ and each $y=0 . \alpha_{1} \alpha_{2} \alpha_{3} \cdots \in g^{*}$ and such that $t_{n}(y)$ $\rightarrow 1 / 2$. Since $\eta^{*}$ is of measure one it contains a subset $\mathfrak{Y}^{* *}$ of measure one such that if $y \in Y^{* *}$ then also $1-y \in Z^{* *}$. Choosing any such $y$ we may write $y=0 . \alpha_{1} \alpha_{2} \alpha_{3} \cdots$ and $1-y=0 . \beta_{1} \beta_{2} \beta_{3} \cdots$, where $\alpha_{k}+\beta_{k}=1$ for all $k$. Then (2.2) follows from the fact that

$$
\sum_{k=1}^{\infty} a_{n, k} \alpha_{k}+\sum_{k=1}^{\infty} a_{n, k} \beta_{k}=\sum_{k=1}^{\infty} a_{n, k} \text {. }
$$

To verify (2.3) we introduce the Rademacher functions $R_{k}(y)$ defined for each 
$k$ and each $y=0 . \alpha_{1} \alpha_{2} \alpha_{3} \cdots \in \in$ as $1-2 \alpha_{k}(y)$. Then

$$
t_{n}(y) \equiv \sum_{k=1}^{\infty} a_{n, k} \dot{v i k}_{k}(y)=\frac{1}{2} \sum_{k=1}^{\infty} a_{n, k}-\frac{1}{2} \sum_{k=1}^{\infty} a_{n, k} R_{k}(y)
$$

must exist almost everywhere in for each $n$. In view of (2.2) the necessity of (2.3) follows from a well-known result of kolmogoroff $[6, \mathrm{p} .126]$.

To establish (2.4) let $k$ be fixed and denote by $\mathfrak{Y}_{1}^{*}$ and $\mathfrak{V}_{2}^{*}$ the subsets of $\mathfrak{V}^{*}$ (defined above) of measure $2^{-k}$ which lie, respectively, in the intervals $0<y$ $<2^{-k}$ and $2^{-k}<y<2^{-k+1}$. It is evident that there exist subsets $\mathfrak{Y}_{1}^{* *}$ of $\mathfrak{Y}_{1}^{*}$ and $\mathfrak{S}_{2}^{* *}$ of $\mathfrak{S}_{2}^{*}$, of measure $2^{-k}$, such that if $y \in \mathbb{V}_{1}^{* *}$ then $y+2^{-k} \in \mathfrak{R}_{2}^{* *}$. For such a value of $y$ we have $y=0.00 \cdots 0 \alpha_{k+1} \alpha_{k+2} \cdots(k+1$ zeros $)$ and $y$ $+2^{-k}=0.00 \cdots 01 \alpha_{k+1} \alpha_{k+2} \cdots(k$ zeros $)$. Consequently, $t_{n}\left(y+2^{-k}\right)-t_{n}(y)$ $=a_{n, k} \longrightarrow 0$ as $n \longrightarrow \infty$.

The proof of the necessity of (2.5) is more involved. Since (2.3) implies the convergence almost everywhere in $\mathscr{Z}$ of the series $\sum_{k=1}^{\infty} a_{n, k} R_{k}(y)$ for each $n$, it follows from Egoroff's theorem that there exists for each $n$ a subset $I_{n}$ of $\mathfrak{S}$ of measure $\left|I_{n}\right|>1-2^{-n-1}$, and an index $\phi_{1}(n)$, increasing to infinity with $n$, such that

$$
\left|\sum_{k=m+1}^{\infty} a_{n, k} K_{k}(y)\right|<\frac{1}{n} \text { for all } m \geq \phi_{1}(n) \text { and all } y \in I_{n} \text {. }
$$

Setting $I=\prod_{n=1}^{\infty} I_{n}$ and using $\mathbb{S}_{E}$ to denote the complement of $E$ with respect to $\mathfrak{Y}$, we have

$$
|s I| \leq \sum_{n=1}^{\infty} \mid\left(s I_{n} \mid<\frac{1}{2}\right.
$$

Consequently we have $|l|>1 / 2$, and (2.7) holds in $I$. We need also the fact that (2.3) insures for each $n$ the existence of an index $\phi(n) \geq \phi_{1}(n)$ for which

$$
\sum_{k>\phi(n)} a_{n, k}^{2}<\frac{1}{n}
$$

Now it follows from (2.2), (2.3), and (2.6) that $T$ ' will have the Borel property if and only if $\tau_{n}(y) \equiv \sum_{k=1}^{\infty} a_{n, k} R_{k}(y)$ approaches zero almost everywhere in $\mathfrak{Z}$ as 
$n \rightarrow \infty$. Writing $\tau_{n}(y)$ in the form

$$
\sum_{k=1}^{\phi(n)} a_{n, k} R_{k}(y)+\sum_{k>\phi(n)} a_{n, k} R_{k}(y)
$$

and using (2.7), we see that $T \in(B P)$ implies the approach to zero almost everywhere in $I$ of

$$
\sigma_{n}(y) \equiv \sum_{k=1}^{\phi(n)} a_{n, k} R_{k}(y) .
$$

Let $E$ be a subset of $l$ with $|E|>0$ on which $\sigma_{n}(y)$ approaches zero uniformly, and let

$$
\sigma_{n, m}(y) \equiv \sum_{k=m}^{\phi(n)} a_{n, k} R_{k}(y)
$$

We can now follow an argument due to Kolmogoroff (for the details see [6, pp.127128] or [4]) and arrive at the inequality

$$
\int_{E} \sigma_{n, \mu}^{2}(y) d y \geq \frac{1}{2}|E| \sum_{k=\mu}^{\phi(n)} a_{n, k}^{2},
$$

for a certain fixed $\mu$ and all $n$ sufficiently large. From (2.4) it follows that

$$
\sigma_{n, \mu}(y)=\sigma_{n}(y)-\sum_{k=1}^{\mu-1} a_{n, k} R_{k}(y)
$$

tends to zero uniformly in $E$ together with $\sigma_{n}(y)$. Then (2.9) yields

$$
\sum_{k=\mu}^{\phi(n)} a_{n, k}^{2}=o(1)
$$

as $n \longrightarrow \infty$. Finally from (2.4) and (2.8) we conclude that

$$
A_{n}=\sum_{k=1}^{\mu-1} a_{n, k}^{2}+\sum_{k=\mu}^{\phi(n)} a_{n, k}^{2}+\sum_{k>\phi(n)} a_{n, k}^{2}=o(1)
$$


as $n \rightarrow \infty$. This completes the proof of Theorem (2.1).

It will be noticed incidentally that conditions (2.2) and (2.4) are among the familiar Silverman-Toeplitz conditions for the regularity of $T$. The remaining condition for regularity, namely,

$$
\sum_{k=1}^{\infty}\left|a_{n, k}\right|=O(1)
$$

is not necessary in order that $T$ have the Borel property. This is shown by the example of the following matrix which appears in $[1]$ :

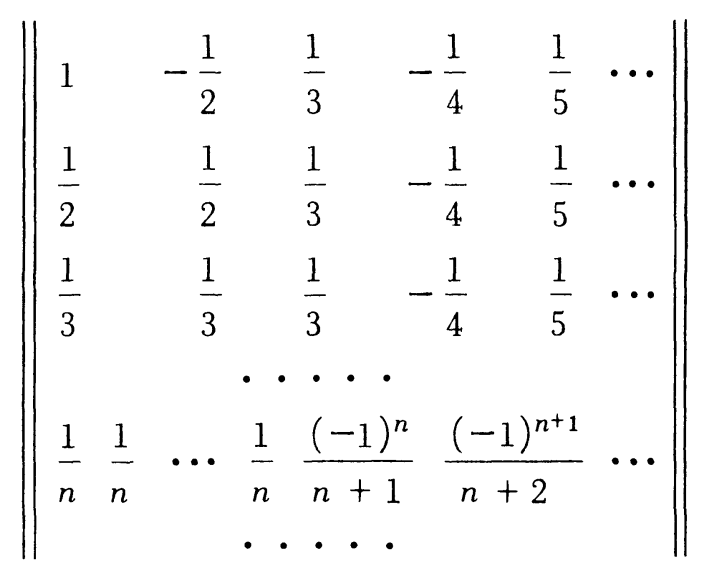

This matrix violates condition (2.10) but satisfies the sufficient conditions of Theorem (3.3) below. It has been proved in [1], however, that $T$ is necessarily regular if it evaluates to $1 / 2$ all sequences of 0 's and l's which are summable$(C, 1)$ to $1 / 2$.

3. Sufficient conditions. We first raise the obvious question of whether the conditions (2.2) and (2.5), which imply (2.3) and (2.4), are sufficient in order that $T \in(B P)$. Before showing that the answer is in the negative, even with the addition of (2.10), we make a few preliminary remarks. Using the notations of $\S_{2}$, and appealing to the Riesz-Fisher theorem, we are led at once to the Parseval relation $\int_{0}^{1} \tau_{n}^{2}(y) d y=A_{n}$. The condition $A_{n} \longrightarrow 0$ is therefore equivalent to the convergence of $\left\{\tau_{n}\right\}$ to zero in the space $L^{2}$, and this assures the existence of 
a sequence of indices $\left\{n_{i}\right\}$ such that $\tau_{n_{i}}(y) \rightarrow 0$ almost everywhere in $\mathfrak{V}$. In other words, if (2.2) and (2.5) are satisfied, the matrix $\left(a_{n, k}\right)$ contains a rowsubmatrix $\left(a_{n_{i}, k}\right)$ defining a method $T^{*}$ (not weaker than $T$ ) having the Borel property; this fact was obtained in [5] with the aid of (2.10). We proceed now to the construction of an example which shows that in the absence of further conditions nothing more can be said.

We need the following result due to Borel [3, pp. 37-47]. The form stated here is less general than the original, in that the groups of consecutive $\alpha$ 's are not permitted to overlap, but it is sufficient for our purposes.

(3.1) Lemma (Borel). Let $\left\{\lambda_{n}\right\}$ be a sequence of positive integers, and let the positive integers $\left\{n_{j}\right\}$ be such that $n_{j} \geq n_{j-1}+\lambda_{j-1}(j=2,3,4, \cdots)$. Then in order that almost all dyadic fractions $y=0 . \alpha_{1} \alpha_{2} \alpha_{3} \cdots$ have the property that for infinitely many $j, \alpha_{n_{j}}$ is followed by $\lambda_{j}$ zeros, and for infinitely many $j$, by $\lambda_{j}$ ones, it is necessary and sufficient that $\sum_{n=1}^{\infty} 2^{-\lambda_{n}}=\infty$.

We can now construct the example of Erdös which was outlined in a letter to the author. The details have been modified to render the matrix triangular but the idea otherwise remains essentially as communicated. We use the notation $a(n, k)$ as alternative to $a_{n, k}$, and define a matrix as follows, wherein, as usual, [log $\left.m\right]$ means the greatest integer in $\log m$. Let

$$
a\left\{\left(m^{2}+i-1\right),\left(\overline{m-1^{2}}+j-2\right)\right\} \equiv[\log m]^{-1}
$$

for $j=i+1, i+2, \cdots, i+[\log m] ; i=1,2, \cdots, 2 m+1 ; m=3,4,5, \cdots$;

and let $a_{n, k} \equiv 0$ otherwise. This matrix of nonnegative terms is evidently triangular, regular, and such that (2.5) is satisfied. On the other hand we have

$$
t_{m^{2}+2 m}(y)=[\log m]^{-1} \sum_{\nu=1}^{[\log m]} a_{m^{2}+\nu}(y) \quad(m=3,4,5, \cdots),
$$

and since $\sum 2^{-[\log m]}=\infty$ it follows from Lemma (3.1) that for almost all $y=$ $0 . \alpha_{1} \alpha_{2} \alpha_{3} \cdots$ there are infinitely many values of $m$ for which $\alpha_{m^{2}}$ is followed by $[\log m]$ zeros, and also infinitely many $m$ for which $\alpha_{m^{2}}$ is followed by $[\log m]$ ones. Hence we see from (3.2) that for almost all $y$ the sequence $\left\{t_{n}(y)\right\}$ contains both infinitely many zeros and infinitely many ones. Consequently the matrix $\left(a_{n, k}\right)$ fails to have the Borel property.

The search for conditions which are necessary as well as sufficient has so 
far yielded no results. However, the sufficient conditions set forth in the following theorems appear to be of interest.

(3.3) THEOREM. In order that $T \in(B P)$, the conditions (2.2) and

$$
\left.\sum_{n=1}^{\infty} A_{n}^{q}<\infty \quad \text { (for some } q>0\right)
$$

are sufficient $[5]$.

Proof. The proof of this theorem given in [5] remains valid under the present weaker conditions. A new criterion involving, as we show later, a condition considerably weaker than (3.4) is contained in the following theorem.

(3.5) THE OREM. In order that $T \in(B P)$ the conditions (2.2) and

$$
\sum_{n=1}^{\infty} \exp \left(-\delta^{2} / 2 A_{n}\right)<\infty \quad(\text { for each } \delta>0)
$$

are sufficient.

For the proof it is convenient to have the following lemma. ${ }^{1}$

(3.7) LEMMA. In order that a sequence $\left\{f_{n}(y)\right\}$ of measurable functions on $\mathbb{2}$ converge to zero almost everywhere it is necessary and sufficient that given $\delta>0$ and $\epsilon>0$ there should exist an index $\nu=\nu(\epsilon, \delta)$ such that

$$
\left|\prod_{n=\nu}^{\infty} E_{n}(\delta)\right|>1-\epsilon,
$$

where $E_{n}(\delta) \equiv E\left\{\left|f_{n}(y)\right| \leq \delta\right\}$.

Proof of Lemma (3.7). Inasmuch as we make no use of the necessity we give only the proof of the sufficiency. Let $\lambda(y)=\varlimsup_{n \rightarrow \infty}\left|f_{n}(y)\right|$, and set $H=E\{\lambda(y)$ $>0\}$. For $m=1,2,3, \cdots$, we set $H_{m}=E\{\lambda(y)>1 / m\}$ so that

$$
H=\sum_{m=1}^{\infty} H_{m} .
$$

1 Added in proof: see P. R. Halmos, Heasure Theory, Van Nostrand, New York, 1950, p.91, Theorem A. 
If $|H|>0$, contrary to the statement of the lemma, then there is an index $\mu$ such that $\left|H_{\mu}\right|>0$. For $\delta=1 / \mu$ and $\epsilon=(1 / 2)\left|H_{\mu}\right|$ the condition (3.8) becomes

$$
\left|\prod_{n=\nu}^{\infty} E_{n}\left(\frac{1}{\mu}\right)\right|>1-\frac{1}{2}\left|H_{\mu}\right|
$$

for an index $\nu=\nu(\mu)$. Consequently

$$
\left|H_{\mu} \cdot \prod_{n=\nu}^{\infty} E_{n}\left(\frac{1}{\mu}\right)\right|>\frac{1}{2}\left|H_{\mu}\right|>0 .
$$

For any point

$$
y_{0} \in H_{\mu} \cdot \prod_{n=\nu}^{\infty} E_{n}\left(\frac{1}{\mu}\right)
$$

we have $\lambda\left(y_{0}\right)>1 / \mu$ since $y_{0} \in H_{\mu}$. On the other hand, since

$$
y_{0} \in \prod_{n=\nu}^{\infty} E_{n}\left(\frac{1}{\mu}\right),
$$

we have $\left|f_{n}\left(y_{0}\right)\right| \leq 1 / \mu$ for all $n \geq \nu$, and this yields $\lambda\left(y_{0}\right) \leq 1 / \mu$. With this contradiction the proof is complete

Proof of (3.5). Proceeding as we did in proving the necessity of (2.5), we first determine an index $\phi(n)$, approaching infinity with $n$, and a set $l$ of positive measure such that

(3.9) $\left|\sum_{k>\phi(n)} a_{n, k} R_{k}(y)\right|<\frac{1}{n}$ for all $y \in I$ and $n=1,2,3, \cdots$.

If we set

$$
B_{n}=\sum_{k=1}^{\phi(n)} a_{n, k}^{2}
$$


then it follows from (3.6) that

$$
\left.\sum_{n=1}^{\infty} \exp \left(-\delta^{2} / 2 B_{n}\right)<\infty \quad \text { (for each } \delta>0\right)
$$

since $B_{n} \leq A_{n}$. Now

$$
\tau_{n}(y)=\sum_{k=1}^{\phi(n)} a_{n, k} R_{k}(y)+\sum_{k>\phi(n)} a_{n, k} R_{k}(y) \equiv \sigma_{n}(y)+\rho_{n}(y),
$$

where $\rho_{n}(y) \longrightarrow 0$ for all $y \in I$, by (3.9). Let $I^{*} \supset I$ denote the entire subset of $\geqslant$ on which $\rho_{n}(y) \rightarrow 0$, so that $\left|I^{*}\right|>0$. If $0 . \alpha_{1} \alpha_{2} \cdots \alpha_{n} \cdots$ is any point of $I^{*}$, it is clear from the definition of $\rho_{n}(y)$ that every point of the form $0 . \beta_{1} \beta_{2} \cdots \beta_{p} \alpha_{p+1} \alpha_{p+2} \cdots$ is likewise in $I^{*}$. Hence $I^{*}$ is a homogeneous set of positive measure, and therefore of measure one (see [9] and [4]). Since $\rho_{n}(y)$ $\longrightarrow 0$ almost everywhere, we complete the proof by showing that (3.10) implies that $\sigma_{n}(y) \longrightarrow 0$ almost everywhere. For this purpose let $E_{n}(\delta)=E\left\{\left|\sigma_{n}(y)\right|\right.$ $\leq \delta\}$ for $\delta>0$. By a lemma of Khintchine [7] we have

$$
\left|\Subset E_{n}(\delta)\right|<M \exp \left(-\delta^{2} / 2 B_{n}\right)
$$

for $n=1,2,3, \cdots$, where $M$ is an absolute constant. Let $\delta>0$ and $\epsilon>0$ be given. Then from (3.10) there exists an index $\nu=\nu(\epsilon, \delta)$ such that

$$
M \sum_{n=\nu}^{\infty} \exp \left(-\delta^{2} / 2 B_{n}\right)<\epsilon
$$

Consequently

$$
\left|\prod_{n=:,}^{\infty} E_{n}(\delta)\right| \geq 1-\sum_{n=\nu}^{\infty} \mid\left(s E_{n}(\delta) \mid>1-\epsilon .\right.
$$

It now follows from Lemma (3.7) that $\sigma_{n}(y) \longrightarrow 0$ almost everywhere.

As a partial consequence of Theorem (3.5) we are able to decide a conjecture of Erdös (made in a letter to the author) to the effect that (2.2) and $A_{n} \log n=o(1)$ are necessary and sufficient in order that $T \in(B P)$.

(3.11) THEOREM. In order that T have the Borel property, the conditions (2.2) 
and

$$
A_{n} \log n=o(1)
$$$$
(n \rightarrow \infty)
$$

are sufficient; but neither (3.12) nor (3.6) is necessary.

Proof. To prove the sufficiency it is enough to show that (3.12) implies (3.6). For this purpose, let $\delta>0$ be given and fix $\epsilon>0$ so that $\delta^{2} / 2 \epsilon>1$. By (3.12) there exists an index $n_{0}$ such that $A_{n}<\epsilon /(\log n)$ for all $n \geq n_{0}$. Then

$$
\exp \left(-\delta^{2} / 2 A_{n}\right)<n^{-\delta^{2} / 2 \epsilon}
$$

for $n \geq n_{0}$ with $\delta^{2} / 2 \epsilon>1$, and (3.6) follows.

To complete the proof we show somewhat more, namely, that no condition of the form $A_{n} \psi(n)=o(1)$, with $\psi(n) \rightarrow \infty$, is necessary. Consequently the Borel property can not be characterized in terms of the rate at which $A_{n}$ approaches zero. For let $0<\theta(n)<1, \theta(n) \rightarrow 0$, with $\theta(n)$ arbitrary otherwise. Let $x_{n}$ $=[1-\theta(n)] /[1+\theta(n)]$, so that $\theta(n)=\left(1-x_{n}\right) /\left(1+x_{n}\right), 0<x_{n}<1$, and $x_{n} \rightarrow 1$. Since the Abel method has the Borel property [5], the same is true of the "discrete" Abel method defined by the matrix

$$
a_{n, k}=\left(1-x_{n}\right) x_{n}^{k-1} \quad(k, n=1,2,3, \cdots) .
$$

For this matrix we find that

$$
A_{n}=\sum_{k=1}^{\infty} a_{n, k}^{2}=\theta(n),
$$

where $\theta(n)$ may tend to zero in any preassigned manner. Thus, for example, if

$$
\theta(n)=\frac{\log \log (n+2)}{\log (n+2)},
$$

we have $A_{n} \log n \longrightarrow \infty$. Finally, if we take $\theta(n)$ as $1 / \log \log (n+p)$, for $p$ sufficiently large, the series in (3.6) diverges for every $\delta>0$.

We now wish to show, as mentioned earlier, that condition (3.4) of Theorem (3.3) implies condition (3.6) of Theorem (3.5), but not conversely. If (3.4) holds for some $q>0$, we have

$$
0<z_{n} \equiv 2 A_{n} / \delta^{2} \rightarrow 0
$$


for each $\delta>0$. Since

$$
\exp \left(-1 / z_{n}\right)=o\left(z_{n}^{q}\right) \text { or } \exp \left(-\delta^{2} / 2 A_{n}\right)=o\left(A_{n}^{q}\right)
$$

as $n \rightarrow \infty$, it follows that (3.6) is satisfied. On the other hand, for the logarithmic method of regular liiesz means defined by

$$
a_{n, k}=1 / k \log (n+1) \text { for } k=1,2, \cdots, n ; n=1,2,3, \cdots \text {, }
$$

we have

$$
A_{n} \cong \pi^{2} / 6 \log ^{2} n
$$

Hence for every $q>0$ the series in (3.4) diverges, but $A_{n} \log n=o(1)$, so that (3.6) holds by the proof of Theorem (3.11).

As a simple application of Theorem (3.11), we call attention to the existence of a regular method having the Borel property and which is weaker than $(C, \alpha)$ for every $\alpha>0$. It suffices to consider the harmonic method $N_{h}$ of regular Nörlund means defined by

$$
a_{n, k}=1 /(n-k+1) \log (n+1) \text { for } k=1,2, \cdots, n ; n=1,2,3, \cdots \text {. }
$$

It is known [8] that $N_{h} \subset(C, \alpha)$ for all $\alpha>0$, and we have here again

$$
A_{n} \cong \pi^{2} / 6 \log ^{2} n
$$

\section{REFERENCES}

1. R. P. Agnew, Methods of summability which evaluate sequences of zeros and ones summable $C_{1}$, Amer. J. Math. 70 (1948), 75-81.

2. É. Borel, Les probabilitiés dénombrables et leurs applications arithmétiques, Rend. Circ. Nat. Palermo 27 (1909), 247-271.

3. - Traité du calcul des probabilitiés et de ses applications, vol. II, part 1, Gautier-Villars, Paris, 1926.

4. R.C. Buck and H. Pollard, Convergence and summability properties of subsequences, Bull. Amer. Math. Soc. 49 (1943), 924-931.

5. J. D. Hill, Summability of sequences of 0's and 1's, Ann. of Math. 46 (1945), 556-562.

6. S. Kaczmarz and H. Steinhaus, Theorie der Urthogonalreihen, Warsaw, 1935.

7. A. Khintchine, ̈̈ber dyadische Brüche, Math. Z. 18 (1923), 109-116.

8. M. Riesz, Sur l'équivalence de certaines méthodes de sommation, Proc. London Math. Soc. (2) i2: (1923), 412-419.

9. C. Visser, The law of nought-or-one, Studia Math. 7 (1938), 143-159. 



\title{
PACIFIC JOURNAL OF MATHEMATICS
}

\section{EDITORS}

\author{
HERBERT BUSEMANN \\ University of Southern California \\ R. M. RoBINSON \\ Los Angeles 7, California \\ University of California \\ Berkeley 4, California \\ E. F. BECKENBACH, Managing Editor \\ University of California \\ Los Angeles 24, California
}

\section{ASSOCIATE EDITORS}

R. P. DILWORTH
HERBERT FEDERER
MARSHALL HALL

\author{
P. R. HALMOS \\ HEINZ HOPF \\ R. D. JAMES
}

\author{
B $\emptyset$ RGE JESSEN \\ PAUL LÉVY \\ GEORGE PÓLYA
}

\author{
J. J. STOKER \\ E. G. STRAUS \\ KÔSAKU YOSIDA
}

\section{SPONSORS}

UNIVERSITY OF BRITISH COLUMBIA

CALIFORNIA INSTITUTE OF TECHNOLOGY

UNIVERSITY OF CALIFORNIA, BERKELEY

UNIVERSITY OF CALIFORNIA, DAVIS

UNIVERSITY OF CALIFORNIA, LOS ANGELES

UNIVERSITY OF CALIFORNIA, SANTA BARBARA

OREGON STATE COLLEGE

UNIVERSITY OF OREGON

\author{
UNIVERSITY OF SOUTHERN CALIFORNIA \\ STANFORD UNIVERSITY \\ WASHINGTON STATE COLLEGE \\ UNIVERSITY OF WASHINGTON

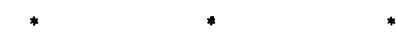 \\ AMERICAN MATHEMATICAL SOCIETY \\ NATIONAL BUREAU OF STANDARDS, \\ INSTITUTE FOR NUMERICAL ANALYSIS
}

Mathematical papers intended for publication in the Pacific Journal of Mathematics should be typewritten (double spaced), and the author should keep a complete copy. Manuscripts may be sent to any of the editors. All other communications to the editors should be addressed to the managing editor, E. F. Beckenbach, at the address given above.

Authors are entitled to receive 100 free reprints of their published papers and may obtain additional copies at cost.

The Pacific Journal of Mathematics is published quarterly, in March, June, September, and December. The price per volume (4 numbers) is $\$ 8.00$; single issues, $\$ 2.50$. Spécial price to individual faculty members of supporting institutions and to members of the American Mathematical Society: $\$ 4.00$ per volume; single issues, $\$ 1.25$.

Subscriptions, orders for back numbers, and changes of address should be sent to the publishers, University of California Press, Berkeley 4, California.

UNIVERSTTY OF CALIFORNIA PRESS • BERKELEY AND LOS ANGELES 


\section{Pacific Journal of Mathematics}

\section{Vol. 1, No. $3 \quad$ BadMonth, 1951}

R. P. Boas, Completeness of sets of translated cosines ............. 321

J. L. Brenner, Matrices of quaternions . ..................... 329

Edmond Darrell Cashwell, The asymptotic solutions of an ordinary differential equation in which the coefficient of the parameter is

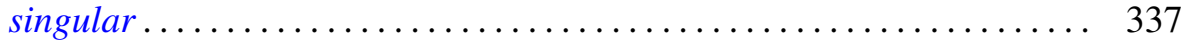

James Dugundji, An extension of Tietze's theorem ................ 353

John G. Herriot, The polarization of a lens ...................... 369

J. D. Hill, The Borel property of summability methods ............... 399

G. G. Lorentz, On the theory of spaces $\Lambda \ldots \ldots \ldots \ldots \ldots \ldots \ldots \ldots \ldots . \ldots \ldots$

J. H. Roberts and W. R. Mann, On a certain nonlinear integral equation of the Volterra type ................................. 431

W. R. Utz, A note on unrestricted regular transformations . . .......... 447

Stanley Simon Walters, Remarks on the space $H^{p} \ldots \ldots \ldots \ldots \ldots \ldots . . \ldots 55$

Hsien Chung Wang, Two theorems on metric spaces ............... 473 